\title{
Optimized Method for Knee Displacement Measurement in Vehicle Sled Crash Test
}

\author{
Hang Sun ${ }^{1, \mathrm{a}}$, Lei Lou ${ }^{1, \mathrm{~b}}$, Zhixin $\mathrm{Liu}^{1}$, Shicheng $\mathrm{Li}^{1}$, Chao Chen ${ }^{1}$ and Xing Pan ${ }^{2, \mathrm{c}}$ \\ ${ }^{1}$ CATARC, China Automotive Technology and Research Center, Tianjin, China. \\ ${ }^{2}$ North Carolina State University Computer Science Department, Raleigh, North Carolina, U.S.

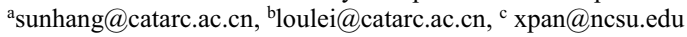

\begin{abstract}
This paper provides an optimized method for measuring dummy's knee displacement in vehicle sled crash test. The proposed method utilizes completely new elements for measurement, which are acceleration and angular velocity of dummy's pelvis, as well as the rotational angle of its femur. Compared with the traditional measurement only using camera-based high-speed motion image analysis, the optimized one can not only maintain the measuring accuracy, but also avoid the disturbance caused by dummy movement, dashboard blocking and knee deformation during the crash. An experiment is made to verify the accuracy of the proposed method, which eliminates the strong dependence on single target tracing in traditional method. Moreover, it is very appropriate for calculating the penetration depth to the dashboard.
\end{abstract}

Keywords. Knee Mapping, vehicle sled test, motion image analysis, dashboard penetration

\section{Introduction}

During the vehicle frontal collision, knee injuries essentially come from the impact between the knee and the dashboard. The material and inner structure of the dashboard have a great influence on the injury level which is evaluated by femur compressive force and knee slider value [1]. Previous researches have shown that the injury level highly depends on the knee penetration against the dashboard and its severity will rise with the increasing depth of that penetration [2]. It was also a statement in Euro-NCAP SLED TEST PROCEDURE 2011 [3] that the manufacture has to provide the evidences for validating the knee mapping test result, which is an additional $20 \mathrm{~mm}$ penetration inside the passenger dashboard [4]. At present, the issue for measuring the knee displacement is fully dependent on computer simulation, which can give out only a virtual result rather than an experimental one. However, the precise result is hard to be achieved in the sled crash experiment because the camera view on the knee could be easily blocked by a lot of matters, such as the dummy's arm and the dashboard. And the pasted tracing target may sometimes deviate from its original position because of strong impact force. Each of these unpredictable issues could disqualify the traditional single target-depended method from measuring the knee displacement correctly, especially for the measurement of penetration.

There is a practical way to calculate the displacement, which is an integral operation conducted twice on acceleration data [5]. Previous researches have made it practical in sled test to measure the displacement of the gravity center of the dummy's head [6], but the situation in measuring knee displacement is much more complicated because the acceleration sensor is hard to be mounted on the knee joint. However, the acceleration data from pelvis-mounted sensor could help to establish the knee displacement [3]. But experiments showed that the results calculated by pelvis acceleration were not very closed to the results using traditional target tracing method, unless the femur rotation around $\mathrm{H}$-point had been taken into account as well. To obtain a precise knee displacement, femur rotaional angle is introduced in this paper, which can help to build a more reasonable way to obtain knee displacement.

\section{Material and Methods}

Traditional Method. In vehicle sled crash test, motion images captured by high-speed camera would be applied to a detailed analysis by engineers [7]. Target tracing measurement is a common analysis method, which is used to calculate the displacements of the parts under test by tracing quadrant symmetry targets [8] pasted on them. Here is the procedure in principle:

- Connecting several pre-determined points on the vehicle to establish a fundamental ruler for the following calculation.

- Establishing a proportion by the lengths of the same ruler in two dimensions, which are the length recorded in length units in the real space and in pixels in the scaled image.

- Establishing a proportion by the depths of the field of the ruler and the target under test. The proportion can reflect the relationship between the ruler length and the displacement of the target [7]. 
- According to the two proportions obtained, the displacement of the target can be calculated by its location change in pixels in different images.

- The velocity, acceleration and other related physical quantities are also available by introducing high-speed camera shutter frequency as a timing variable.

According to the procedure mentioned above, the dummy's knee displacement can be measured by following steps in vehicle sled test:

Step 1: Choosing two points $\left(S_{1}\right.$ and $S_{2}$ in Fig. 1(a)) on the vehicle in the condition that the line segment connecting them should be parallel to the ground plane. Then, the line segment could be considered as a ruler which defines a coordinate system in both direction and length unit.

Step 2: Pasting quadrant symmetry target right on the knee joint which is under test (Target K).

Step 3: Measuring the depths of the field of ruler $\left(\mathrm{d}_{1}\right)$ and $\mathrm{K}\left(\mathrm{d}_{2}\right)$ to the onboard camera lens.

Step 4: Recording the time $\left(\mathrm{t}_{0}\right)$ at the contact moment between the knee and the dashboard by high speed video analysis or contact detector.

Step 5: Using image analysis to plot a knee displacement curve vs. time as is shown in Fig. 1(b).

Step 6: The knee penetration could be calculated by $f\left(t_{m}\right)-f\left(t_{0}\right)$, in which the $t_{\mathrm{m}}$ is the time that the knee comes to its maximum penetration.

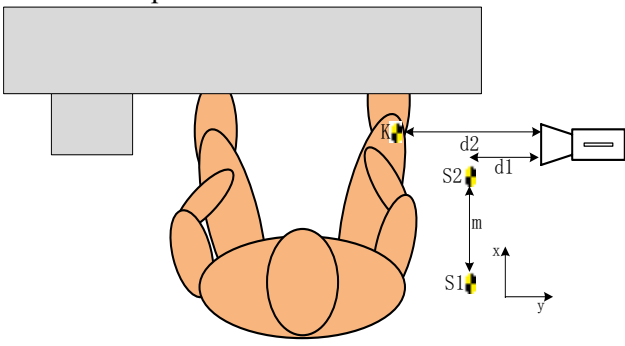

(a) testing setup

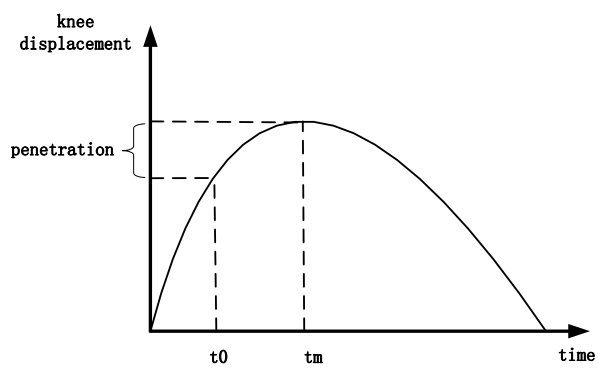

(b) Profile of knee displacement

Fig.1. Knee penetration measured by high-speed motion image analysis.

Proposed Method. In vehicle sled crash test, acceleration and angular velocity sensors can be mounted in the dummy's pelvis in accordance with the position of its H-point. The displacement of H-point along X-axis can be calculated firstly projecting the acceleration to $\mathrm{X}$-axis according to the pelvis angle and then integrating the acceleration to displacement. Dummy femur is a rigid body connecting pelvis with a hinge joint and its length could be considered unchangeable during the crash. So it is possible to use the displacement of pelvis to deduce knee displacement, and its penetration depth as well. But because of the counterforce coming from the impact of the knee against the dashboard, the femur would naturally perform a rotation around H-point, making the displacement of knee less than that of pelvis. And it also brings out the fact that pelvis itself is not enough to represent the knee movement, while an additional revision should be made upon the pelvis displacement to ensure a precise result of knee displacement. The method is proposed as follows:

The orientation of sensors mounted in the pelvis is the same as the pelvis angle. As is shown in Fig. $2, \mathrm{X}_{0}$ and $\mathrm{Z}_{0}$ indicate the sensor coordinate system, which has an angle $\theta$ with the ground coordinate system.

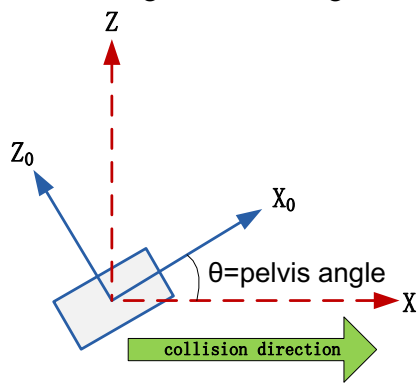

Fig. 2. The mount orientation of the pelvis acceleration sensor.

In order to calculate the displacement of pelvis, the pelvis acceleration should be projected to ground $\mathrm{X}$-axis:

$A C C_{x}=A C C_{X 0} \cdot \cos \theta-A C C_{Z 0} \cdot \sin \theta$

The angle $\theta$ changes over time during the whole process of crash, which could be calculated by integration of angular velocities:

$$
\theta=\int_{0}^{t} \omega(x) d x
$$


Similarly, the displacements of pelvis and sled could be both calculated by integration of their accelerations, and the relative displacement could be figured out by a subtraction of their absolute displacements:

$\mathrm{d}_{\text {pelvis }}=\int_{0}^{t} \int_{0}^{u} A C C_{\text {pelvis }}(x) d x d u-\int_{0}^{t} \int_{0}^{u} A C C_{\text {sled }}(x) d x d u$

In practical test, the data captured by real-time data acquisition device are all discrete values at each sampling moment $\Delta \mathrm{t}$ (reciprocal of sample frequency), which turns the operation of integration into accumulation:

$\theta_{\mathrm{n}}=\sum_{0}^{n} \frac{\omega_{n}+\omega_{n+1}}{2} \cdot \Delta \mathrm{t} \quad(\mathrm{n}=0,1,2 \ldots)$

$$
\begin{aligned}
& \mathrm{d}_{\mathrm{n}}=\sum_{0}^{n} \frac{\sum_{0}^{n} \frac{A C C_{n}+A C C_{n+1}}{2} \cdot \Delta \mathrm{t}+\sum_{0}^{n+1} \frac{A C C_{n+1}+A C C_{n+2}}{2} \cdot \Delta \mathrm{t}}{2} \cdot \Delta \mathrm{t}- \\
& \sum_{0}^{n} \frac{\sum_{0}^{n} \frac{\text { ACCsled }_{n}+\text { ACCsled }_{n+1}}{2} \cdot \Delta \mathrm{t}+\sum_{0}^{n+1} \frac{\text { ACCsled }_{n+1}+\text { ACCsled }_{n+2}}{2} \cdot \Delta \mathrm{t}}{2} \cdot \Delta \mathrm{t} \quad(\mathrm{n}=0,1,2 \ldots)
\end{aligned}
$$

Figure 3 illustrates the knee penetration process. At the contact time $\left(\mathrm{t}_{\mathrm{c}}\right), \alpha$ is defined as the angle between $\mathrm{X}$-axis and $1_{\mathrm{F}}\left(\mathrm{a}\right.$ line segment connecting joints of $\mathrm{H}$-point and knee). And $\beta$ is defined as the angle between $\mathrm{X}$-axis and $\mathrm{l}_{\mathrm{F}}$ at the time $\left(\mathrm{t}_{\mathrm{MAX}}\right)$ that the knee reaches its maximum penetration.

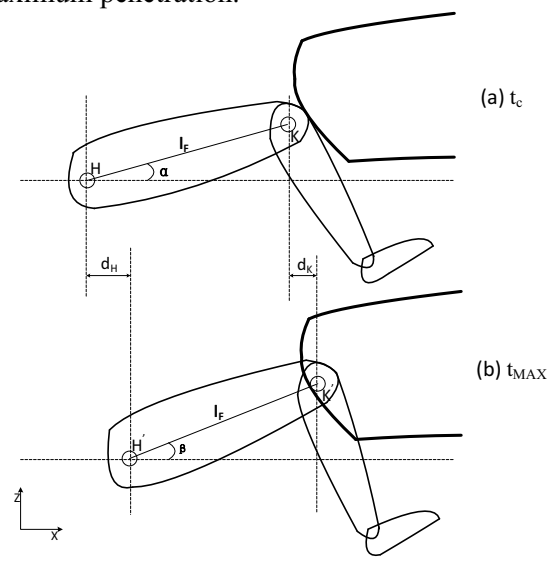

Fig. 3. Penetration measured by proposed method.

The penetration of knee is set for $d_{K}$, and the H-point displacement along X-axis is set for $d_{H}$, which is already worked out through Eq. 5. According to triangle relationship, the penetration can be represented as:

$$
d_{K}=d_{H}-(\cos \alpha-\cos \beta) \cdot l_{F}
$$

\section{Results}

During the whole crash process, the measurement of knee displacement in penetration phase is much more difficult than that of knee displacement in other phases. A comparison is made between two methods provided above based on vehicle sled crash test and a thorough analysis is conducted upon the testing results. By use of accaleration sled facility, the test precisely simulated the crash pulse recorded in full scale vehical frontal collision. In order to achieve a significant depth of the penetraion for better comparison, a Hybrid $95^{\text {th }}$ percentile dummy was uesd in the test with necessary instruments and high-speed cameras were set for recording. And the sample frequency was set to $\mathrm{f}=10^{4}$ to ensure the integral precision. In addition, the arm and hand were all set on top of the femur rather than beside it and the target was pasted on the nearest point of the knee joint for a better camera view. Fig. 4 illustrates the knee displacement during the entire process of crash by the method of single target tracing. The displacement is $30.78 \mathrm{~mm}$ at contact moment $(40 \mathrm{~ms})$, and its maximum is $140.74 \mathrm{~mm}$ at time of $101 \mathrm{~ms}$. So the penetration is $109.96 \mathrm{~mm}$ that can be calculated by a subtraction operation. 


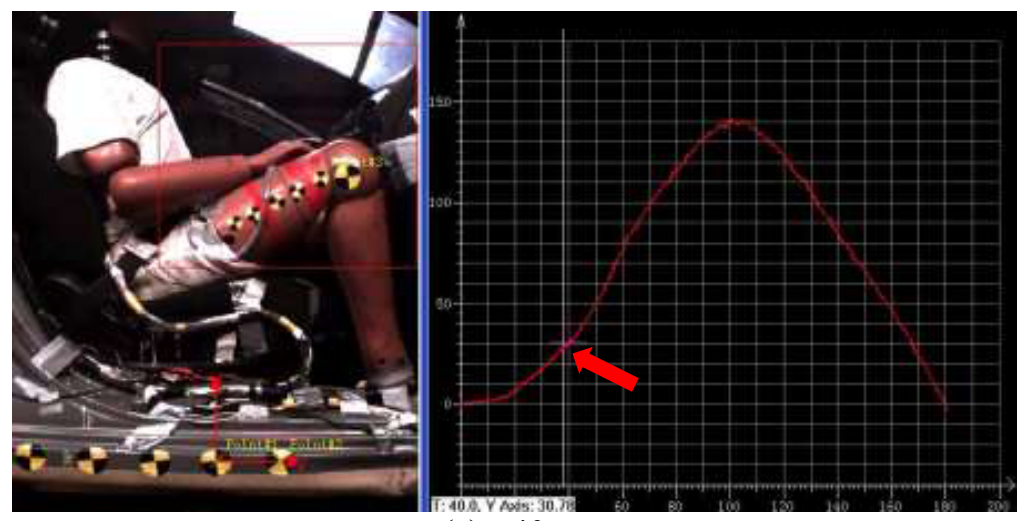

(a) $\mathrm{t}=40 \mathrm{~ms}$
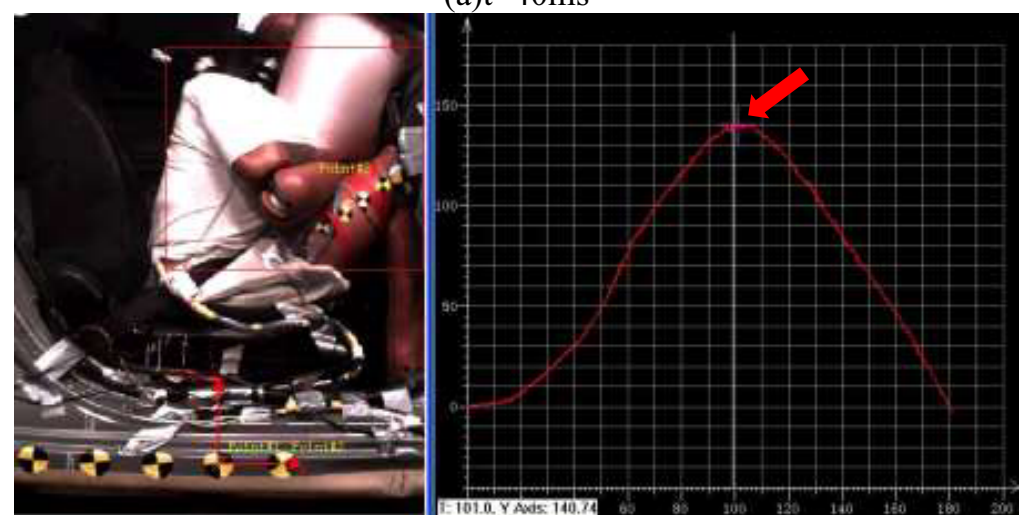

(b) $\mathrm{t}=101 \mathrm{~ms}$

Fig. 4. Knee displacement curve worked out by single target tracing.

Figure 5 illustrates the pelvis acceleration curve based on the data captured by the sensor with its own cordinate system $\mathrm{X}_{0}$ and $\mathrm{Z}_{0}$. The pelvis angle to the horizontal is calculated by integration of the angular velocity captured by angular sensor, which is illustrated in Figure 6 that indicates the changing trend of pelvis angle during the crash. According to the Eq. 1, the pelvis displacement along X-axis is shown in Figure 7(a), and the displacement curve of sled is shown in Figure 7(b) which is original in line with the same X-axis.

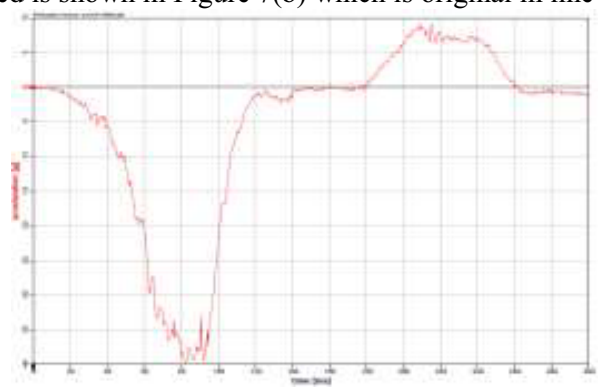

(a) $\mathrm{X}_{0}$

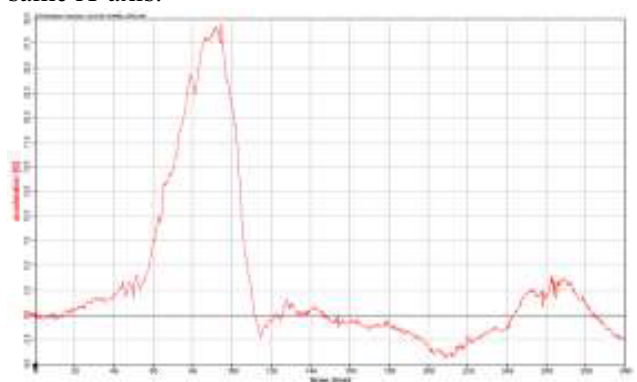

(b) $Z_{0}$

Fig. 5. Curves of original pelvis acceleration. 


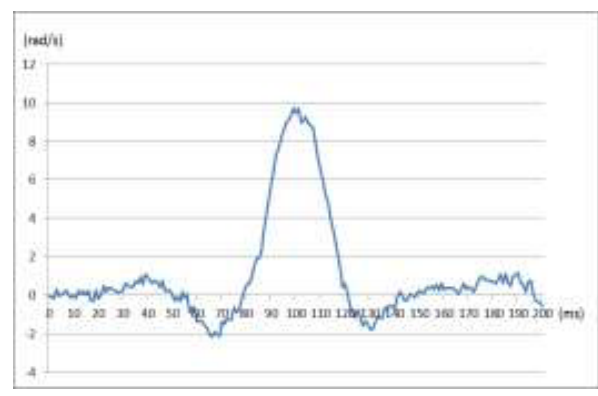

(a) Angular velocity

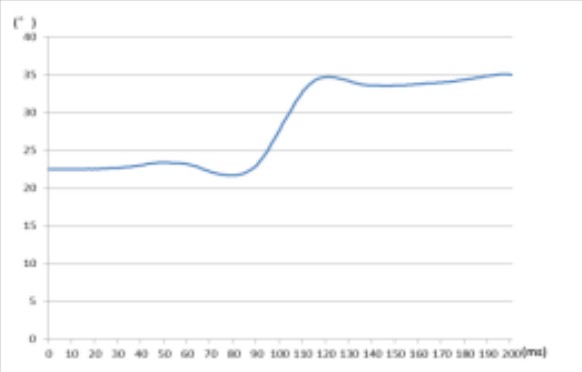

(b) Angle degree

Fig. 6. Curves related to pelvis angle.

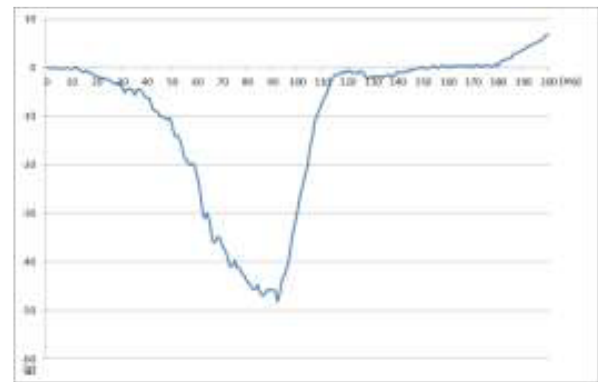

(a) Pelvis

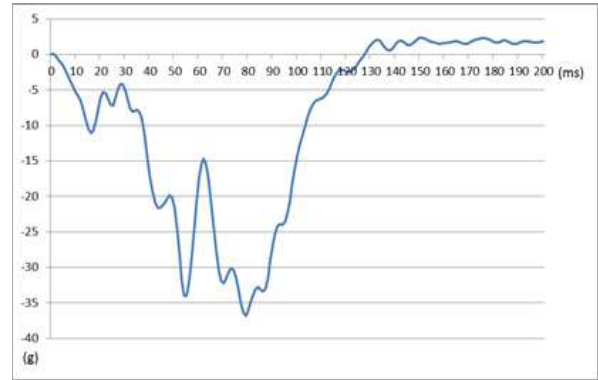

(b) Sled

Fig. 7. Curves of crash displacement

The relative dispalcement in Figure 8 can be calculated by Eq. 5. And the pelvis displacement is $132.19 \mathrm{~mm}$ between the contact time $(40 \mathrm{~ms})$ and the time $(94 \mathrm{~ms})$ when pelvis reaches its peak point of displacement. Without considering femur angle change, there is a $22.23 \mathrm{~mm}$ deviation to the previous result.

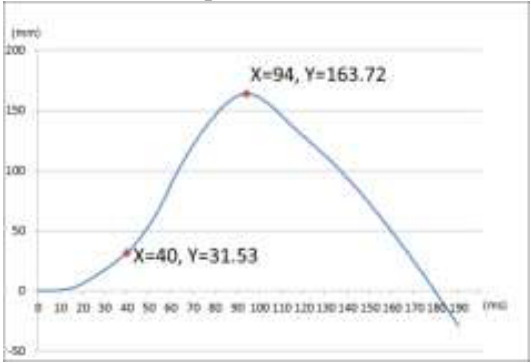

Fig. 8. Knee displacement curve worked out by proposed method

The femur angle changes from $27.19^{0}$ to $34.18^{0}$ shown in Figure 9. And the length between knee joint and H-point is $426.46 \mathrm{~mm}$ [9], so the knee penetartion depth is $105.66 \mathrm{~mm}$ calculated by Eq. 6 .
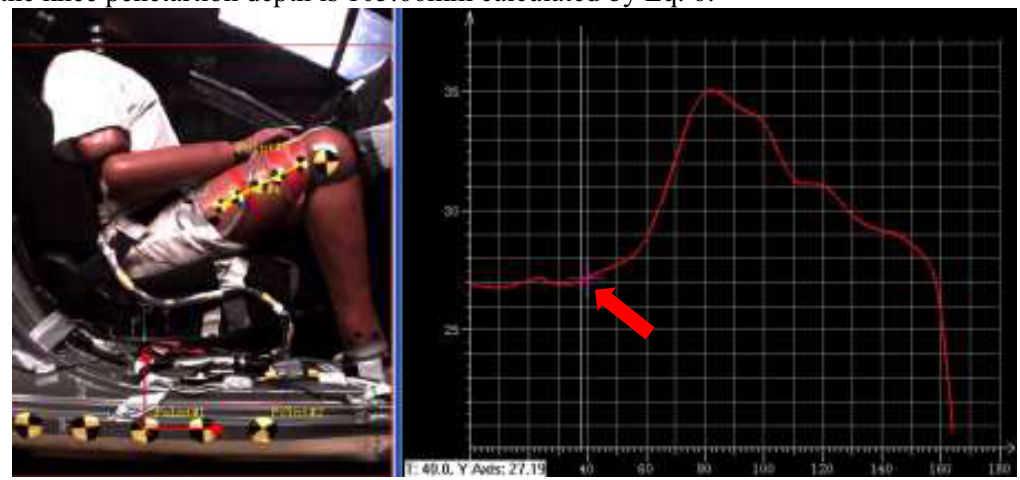

(a) $40 \mathrm{~ms}$ 

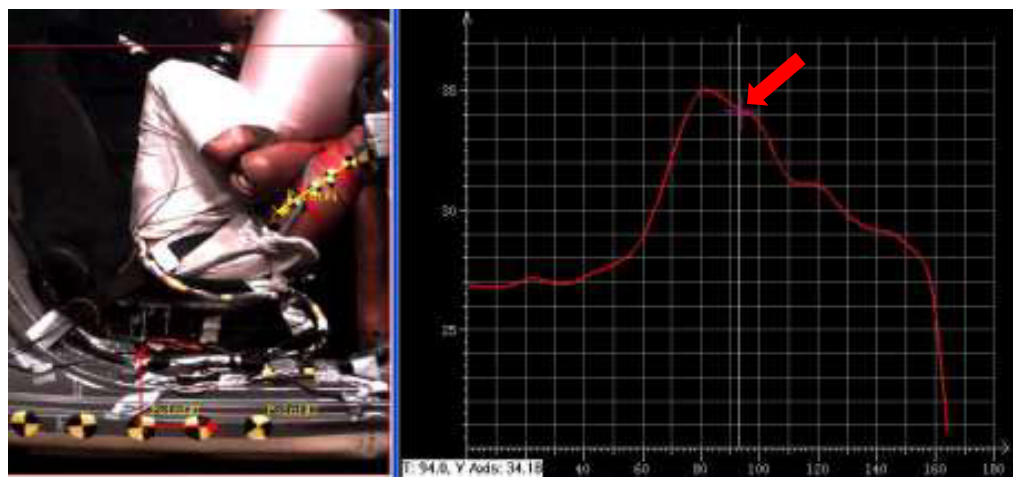

(b) $94 \mathrm{~ms}$

Fig. 9. Femur angle in two particular points of time.

\section{Discussion}

As shown in the results, the femur rotation establishes a correlation between the movement of pelvis and knee and revises the pelvis displacement $132.19 \mathrm{~mm}$ to the knee displacement $105.66 \mathrm{~mm}$, which means a $26.53 \mathrm{~mm}$ difference caused by femur rotation. Compared with the result of $109.96 \mathrm{~mm}$ from single target tracing method, the proposed method has a difference of $4.3 \mathrm{~mm}, 4 \%$ out of the traditional method, which means the results from both methods are basically identical. In addtion, because of the difference in rebound timing, there is a $7 \mathrm{~ms}$ mismatch between the knee and pelvis reaching their maximum displacements respectively (Figure 4 \& Figure 8). But it barely affects the final result because the knee is relatively stationary to the dashboard during that extremely short period.

\section{Summary}

Since the cost for vehicle collision test is significantly considerable, it would be a great risk if the knee displacement measuring is only dependent on single target, which can be easily blocked by several unexpected factors. The proposed method takes advantage of the pelvis acceleration and the femur angle which can be credibly obtained by connecting any two separate targets, which eliminates the dependence on single target. Additionally, since the result of proposed method is quite similar to that of traditional method, the proposed method can be considered as an alternative way to measure the knee displacement, especially effective for knee penetration measurement. In conclusion, the proposed method is applicable to knee impact test and would be very useful in validating knee mapping test.

\section{References}

1. Gokhale A V, Saravate V B, Chalipat S, et al: Femur and knee injury reduction by use of knee bolsters in frontal crashes. Papers. Automotive_Sector (2007).

2. Laurent Portier, Xavier Trosseille: Lower Leg injuries in Real-world Frontal Accidents. IRCOBI Conference (1993).

3. Sled Test Procedure for Assessing Knee Impact Areas (Euro-NCAP 2011-06).

4. Chen Tangpei, Lei Peng: The Study of Knee Mapping Sled Test. The 13th Conference of Automotive Safety Technology (2013).

5. Rong Tianping: The theory and analysis of displacement measuring by acceleration. Journal of Huazhong University of Science and Technology (2000).

6. Lei Peng, Chen Tangpei. A Method of Obtained Dummy Head Displacement by Acceleration and High Speed Camera during the sled test. The 16th International Conference of China Automotive Safety Technology (2013).

7. Li Shicheng, Lou Lei: Research on Parallax Correction Method for High-Speed Image Motion nalysis. Mechanical Research \& Application (2014-01).

8. User Guide of TEMA (2008).

9. User's Manual for the Hybird III Large Male (2003). 